\title{
Derivation of two human embryonic stem cell lines form discarded blastocysts and maintained in conditioned media from human foreskin fibroblasts feeder cells without serum
}

Yonghua Jiang ${ }^{1}$, Xiaofang Sun ${ }^{1}$, Xiaolin Long ${ }^{1}$, Hongzi Du ${ }^{1}$, Xinjie Chen ${ }^{1}$, Yifei Yin ${ }^{1}$, Shengchang Huang ${ }^{1}$,Weihua Wang ${ }^{1}$, Guohong Xiao ${ }^{1}$

${ }^{1}$ Institute of Gynecology and Obstetrics, The Third Affiliated Hospital of Guangzhou Medical College, Duobao Road, Guangzhou, China

In IVF clinics, many poor quality human embryos have been discarded because they showed no survival characteristics at the end of culture and the implantation rate is very low if poor quality embryos are transferred. This study was designed to investigate an effective method for the derivation of human embryonic stem cells (hESC) from poor quality embryos discarded from in vitro fertilization (IVF) laboratories,maintain the hES cell without feeder and serum, minimize exposure to animal cells and proteins. blastocysts used in this study from IVF discarded embryo, ICM isolated by immunosurgery, then placed on mitomycin C-treated MEF feeder layers, after 9 passage, hES cell maintained in conditioned media without Serum,collect conditon medium from human forskins fibroblasts feeder, add $8 \mathrm{ng} / \mathrm{ml}$ bFGF befoure use.human embryonic stem cell colonies were subsequently characterized by cell surface marker staining, karyotyping and teratoma formation. We derive two new hESC lines form discarded blastocysts, the two cell line can maintained in conditioned medium from human foreskin fibroblasts feeder cell with $\mathbf{2 0 \%}$ knockout serum replacement. express several pluripotency markers including alkaline phosphatase, SSEA- 4, Tra-1-60, Tra 1-60,Tra 1-81, Oct-4,GAPDH-R, and SOX2-F, remain in undifferentiated state with normal karyotype after prolonged passages and can form embryoid bodies in vitro and teratoma in vivo.

Keywords: embryonicstem cells, conditioned media, human foreskin fibroblasts, feeder free, serum free

Cell Research (2008) 18:s42. doi: 10.1038/cr.2008.132; published online 4 August 2008

Correspondence: Xiaofang Sun

E-mail: xiaofangsun@hotmail.com 